Revista Brasileira de Agricultura Irrigada v.1, n.1, p.3-8, 2007

Fortaleza, CE, INOVAGRI - http://www.inovagri.org.br Protocolo 005.07 - 16/09/2007

Aprovado em 17/11/2007

\title{
Recomendação de Equações para Determinação da Perda de Carga em Filtros de Tela
}

\author{
Cleomar Ferreira de Oliveira $^{1}$, José Antônio Frizzone ${ }^{2}$ e Osvaldo Rettore Neto ${ }^{3}$
}

\begin{abstract}
RESUMO
Os sistemas de irrigação localizada requerem, obrigatoriamente, sistemas de filtração para o tratamento mínimo da água. Os filtros são fundamentais à qualidade da água utilizada na agricultura irrigada, promovendo melhor desempenho dos sistemas de irrigação e seus componentes. A determinação da perda de carga, devido à instalação de filtros no cabeçal de controle, assume um papel importante no que se refere ao dimensionamento hidráulico do sistema, nesse aspecto, duas informações são relativamente importantes: a perda de carga provocada pela instalação do filtro e pela passagem do fluxo de água através do elemento filtrante no processo de filtragem, enquanto a primeira informação permitiria um melhor dimensionamento do cabeçal de controle, a segunda possibilita um cálculo mais rigoroso da pressão no início da linha de derivação. Dentro deste contexto e com o objetivo de oferecer embasamento técnico para o dimensionamento de um sistema de filtragem com baixo custo e alto nível tecnológico, foram desenvolvidas equações matemática de crescimento potencial da perda de carga em função da vazão circulante em filtros de tela. Os ensaios foram realizados no laboratório de irrigação do Departamento de Engenharia Rural da ESALQ/USP, utilizando-se filtros comerciais da marca AMIAD, com elementos filtrantes de tela (modelos 3/4", 1', $1^{1 / 2 "}$ e 2") mesh 120. A vazão máxima utilizada no ensaio, para cada modelo de filtro, foi à recomendada pelo fabricante. Desta maneira recomenda-se a sua utilização para dimensionamento de projetos hidráulicos.
\end{abstract}

Palavras-chave: Filtração, Qualidade da água, Agricultura irrigada

\section{Recommendation of Equations For Determining Load Losses On Screen Filters}

\begin{abstract}
Drip irrigations systems always require filtration systems for the minimum treatment of water. These filters are fundamental for the water quality used in the irrigated agriculture, promoting better performance of overhead irrigations and their components. The determination of load loss, due to installation of filters in the control bolster, plays an important role in hydraulic system design, in this respect, two aspects are relatively important: The load loss provoked by the installation of the filter and water influx passing through the filter in the process of filtration and allows better control bolster design and permits a more rigorous calculation of the pressure in the beginning of the derivation line. It's in this context and with the objective to offer technical advice for the design of low cost filtration systems with high technological level that mathematical equations for potential increase of head loss as a function of circulating discharge in screen and disks filters were developed. The experiments were carried out at in the laboratory of irrigation in the Department of Rural Engineering of ESALQ/USP, using commercial filters of the mark AMIAD, with screen filter elements (models $3 / 4$ ", 1 ', $1^{1 / 2 "}$ and 2 ") mesh 120. The experiments were done using maximum discharge for each filter model as recommended by the manufacturer, Based on the obtained results, these equations may be used for hydraulic system design for they are simple and reliable trustful.
\end{abstract}

Key words: Filtration, Quality of the water, Irrigated agriculture

\footnotetext{
${ }^{1}$ Doutorando em Irrigação e Drenagem da ESALQ/USP, E-mail : cleomar@esalq.usp.br

${ }^{2}$ Prof. Titular Departamento de Eng. Rural, ESALQ/USP, e-mail: frizzone@esalq.usp.br .

${ }^{3}$ Mestrando em Irrigação e Drenagem da ESALQ/USP, E-mail : orettore@esalq.usp.br
}

R. Bras. Agric. Irrigada, v.1, n.1, p.3-8, 2007. 


\section{INTRODUÇÃO}

De acordo com Caixeta (1991), o termo perda de carga é usado como sendo parte da energia potencial de pressão e de velocidade que é transformada em outros tipos de energia dissipada no processo, tal como calor.

A eficiência do sistema de irrigação está relacionada à qualidade dos seus componentes e ao correto dimensionamento hidráulico do sistema, principalmente no que se refere aos cálculos de perda de carga. $\mathrm{O}$ processo de perda de carga localizada em conexões e peças especiais é bastante complexo, envolvendo variáveis como sua geometria e o processo de fabricação, o regime de escoamento e a presença de correntes secundárias, entre outras (CABELLO, 1996).

Diversas relações matemáticas têm sido produzidas e utilizadas nos cálculos de perda de carga em tubulações, com maiores ou menores restrições de seu coeficiente por parte dos profissionais da área. No caso de peças especiais, com filtros de tela e de discos, cujas perdas de cargas são denominadas de localizadas, o uso de equações gerais torna-se mais difícil, em virtude de suas próprias características e de fenômenos observados em seu interior quando da passagem do líquido. Segundo Testezlaf e Ramos (1995) o modelo alométrico linear é o que melhor explica o fenômeno físico e que demonstra um valor significativo de correlações entre perda de carga e vazão dos filtros de tela e de discos.

A instalação de filtros, em sistemas de irrigação localizada, com objetivo de evitar o entupimento dos emissores na linha de irrigação, produz uma variação no comportamento hidráulico do sistema ao longo do tempo. À medida que a vazão, fornecida ao sistema, atravessa os filtros, provoca a diminuição da filtragem, visto que o material sólido em suspensão se agrega ao corpo do elemento filtrante, formando uma superfície de partículas sólidas na parte superior da areia e na parede dos filtros de tela e de discos. acarretando a elevação da perda de carga e a redução da vazão disponível no filtro, influenciando na altura manométrica e na vazão do sistema.

Matsuda; Testezlaf e Almeida Neto (1989), avaliando a perda de carga em filtros de areia e de tela observou que a variação da altura de areia afeta a perda de carga nos filtros de areia e de tela individualmente, mas não causa variação significativa na perda de carga total. Verificou-se ainda que a perda de carga nos filtros de tela foi sempre maior do que nos filtros de areia com variação de pressão. As curvas indicam que as perda de carga no filtro de areia tem crescimento linear, enquanto a do filtro de tela é exponencial, comprovando uma baixa eficiência de remoção de resíduos ao longo do tempo para os filtros de areia. Os mesmos autores afirmam que a intersecção das curvas de perda de carga dos filtros de areia e de tela representa o ponto a partir do qual a eficiência do filtro de areia é nula. Portanto este ponto pode ser recomendado como referência no uso da retrolavagem dos filtros.

A filtragem tradicional da água na irrigação localizada, em que se utlizam os filtros de tela mostra um campo ideal de discussão, visto que se relaciona a uma capacidade de retenção de matéria. Como forma de gerar subsídios para uma discussão sobre a capacidade de filtragem para esses sistemas foram realizados ensaios, em laboratório, para desenvolver equações matemáticas que estimem a perda de carga localizada nos filtros de tela.

\section{MATERIAL E MÉTODOS}

O experimento foi conduzido no Laboratório de Irrigação do Departamento de Engenharia Rural, da Escola Superior de Agricultura "Luiz de Queiroz" - ESALQ/USP, Campus de Piracicaba - SP. Foram analisadas as perdas de carga, em função da vazão circulante, em filtros de tela e de discos empregados em irrigação localizada, utilizando-se água limpa.

Utilizou-se no experimento medidor de vazão magnético indutivo, com precisão de $1 \%$, manômetro diferencial com coluna de mercúrio e registros.

A perda de carga nos filtros, registrada pelo manômetro diferencial, foi obtida para um conjunto de, no mínimo, 28 valores de vazão, para o filtro de malha de 3/4" e no máximo, 61 valores de vazão para o filtro de discos de 1". A vazão máxima utilizada no ensaio, para cada modelo de filtro, foi a recomendada pelo fabricante, conforme se apresenta na Tabela1. Definiu-se um modelo matemático não linear para descrever a variação da perda de carga 
nos filtros em função da vazão circulante, conforme expresso pela eq. (1).

$$
\mathrm{H}_{\mathrm{f}}=\beta_{0} \mathrm{Q}^{\beta_{1}} \varepsilon
$$

sendo:

$\mathrm{H}_{\mathrm{f}}$ - perda de carga no filtro, em mca;

$\mathrm{Q}$ - vazão que escoa através do filtro, $\mathrm{em}^{3} \mathrm{~h}^{-}$ ${ }^{1}$;

$\beta_{0}$ - constante que expressa a perda de carga para a vazão unitária, em mca;

$\beta_{1}$ - constante característica do regime de fluxo, adimensional;

$\varepsilon$ - erro aleatório.

O modelo potencial, expresso pela eq. (1), é chamado de intrinsecamente linear, uma vez que pode ser linearizado por uma transformação logarítmica, conforme eq. (2).

$$
\ln H_{\mathrm{f}}=\ln \beta_{0}+\beta_{1} \ln \mathrm{Q}+\ln \varepsilon
$$

sendo ln o operador do logaritmo natural. Essa transformação requer que os termos transformados do erro $\ln \varepsilon$ sejam normal e independentemente distribuídos com média 0 e variância $\sigma^{2}$. Dessa forma, o modelo linear se transforma em linear, conforme eq. (3), e a análise estatística pode ser realizada sob os princípios da regressão linear.

$$
Y=\beta+\beta_{1} X+\phi
$$

sendo $Y=\ln H_{f}, \beta=\ln \beta_{0}, X=\ln Q$ e $\phi=\ln \varepsilon$.

O método dos mínimos quadrados foi utilizado para estimar os parâmetros $\beta, \beta_{0}$ e $\beta_{1}$, de forma que estimadores desses parâmetros é dado pelas eq. (4) (5) e (6).

$$
\hat{\beta}_{1}=\frac{\sum_{i=1}^{n} X_{i} Y_{i}-\frac{\sum_{i=1}^{n} X_{i} \sum_{i=1}^{n} Y_{i}}{n}}{\sum_{i=1}^{n} X_{i}^{2}-\frac{\left(\sum_{i=1}^{n} X_{i}\right)^{2}}{n}}
$$

sendo $\mathrm{n}$ o número de pares de pontos vazãoperda de carga, e

R. Bras. Agric. Irrigada, v.1, n.1, p.3-8, 2007.

$$
\begin{aligned}
& \hat{\beta}=\bar{Y}-\beta_{1} \bar{X} \\
& \hat{\beta}_{0}=\operatorname{ant}(\ln \hat{\beta})=\mathrm{e}^{\hat{\beta}}
\end{aligned}
$$

sendo $\underline{\mathrm{e}}$ a base dos logaritmos naturais.

Para determinar a existência de relação significativa entre a variável dependente $\left(\mathrm{H}_{\mathrm{f}}\right)$ e a variável explicativa, ou independente $(\mathrm{Q})$, foram formuladas as seguintes hipóteses:

$\mathrm{H}_{0}: \beta_{1}=0$ (não existe relação potencial entre $\mathrm{H}_{\mathrm{f}}$ e Q)

$\mathrm{H}_{1}: \beta_{1} \neq 0$ (existe relação potencial entre $\mathrm{H}_{\mathrm{f}} \mathrm{e}$ Q)

A hipótese nula $\left(\mathrm{H}_{0}\right)$ foi testada com a estatística $\mathrm{F}$, ao nível de significância de $1 \%$, conforme se indica na Tabela 6 . O teste $\mathrm{F}$ é utilizado para testar razão entre duas variâncias. Quando se testa a significância da regressão, a medida do erro aleatório é chamada variância do erro, de modo que o teste $\mathrm{F}$ é a fração da variância decorrente da regressão dividida pela variância do erro: Assim define-se: a estatística $\mathrm{F}$ é igual ao quadrado médio devido à regressão $\left(\mathrm{QM}_{\mathrm{reg}}\right)$ dividido pelo quadrado médio devido à variação residual $\left(\mathrm{QM}_{\mathrm{res}}\right)$ :

Tabela 1 - Características de fabricação dos filtros utilizados no experimento

\begin{tabular}{lc|c|cc|}
\hline Modelo & $\begin{array}{c}\mathrm{N}^{\mathrm{o}} \\
\text { de } \\
\text { mesh }\end{array}$ & $\begin{array}{c}\text { Vazão } \\
\text { máxima } \\
\text { recomendada } \\
\left(\mathrm{m}^{3} \mathrm{~h}^{-1}\right)\end{array}$ & $\begin{array}{c}\text { Diâmetro de } \\
\text { entrada/saída } \\
(\mathrm{mm})\end{array}$ & $\begin{array}{c}\text { Perda de } \\
\text { carga } \\
\text { especificada } \\
\text { (mca) }\end{array}$ \\
\hline $\begin{array}{l}\text { Tela } \\
\text { 3/4" }\end{array}$ & 120 & 3 & 20 & 2,638 \\
Tela 1" & 120 & 7 & 25 & 5,487 \\
Tela 1 & 120 & 15 & 40 & 3,719 \\
$\begin{array}{l}\text { 1/2" } \\
\text { Tela 2" }\end{array}$ & 120 & 25 & 50 & 2,479 \\
Fonte: AMIAD (2004) & & &
\end{tabular}

Tabela 2 - Análise de variância para testar a significância da regressão

\begin{tabular}{lcccc}
$\begin{array}{l}\text { Fontes de } \\
\text { variação }\end{array}$ & $\begin{array}{c}\text { Soma dos } \\
\text { quadrados }\end{array}$ & $\begin{array}{c}\text { Graus de } \\
\text { liberdade } \\
(\mathrm{GL})\end{array}$ & $\begin{array}{c}\text { Quadrado } \\
\text { médio }\end{array}$ & $\mathrm{F}$ \\
Regressão & $\mathrm{SQ}_{\mathrm{R}}=\hat{\beta}_{1} \mathrm{~S}_{\mathrm{Xy}}$ & 1 & $\mathrm{QM}_{\mathrm{R}}=\mathrm{SQ}_{\mathrm{R}} / \mathrm{GL}$ & $\mathrm{QM}_{\mathrm{R}} / \mathrm{Q}$ \\
Erro & $\begin{array}{c}\mathrm{SQ}_{\mathrm{E}}=\mathrm{SQ}_{\mathrm{T}}- \\
\hat{\beta}_{1} \mathrm{~S}_{\mathrm{Xy}}\end{array}$ & $\mathrm{n}-2$ & $\mathrm{QM}_{\mathrm{E}}=\mathrm{SQ}_{\mathrm{E}} / \mathrm{GL}$ & \\
& & & \\
Total & $\mathrm{SQ}_{\mathrm{T}}=\mathrm{SQ}_{\mathrm{R}}+\mathrm{SQ}_{\mathrm{E}}$ & $\mathrm{n}-1$ & & \\
\hline \multicolumn{2}{l}{ Sendo: $\mathrm{S}_{\mathrm{xy}}$ é o numerador da eq. (4). }
\end{tabular}

A regra de decisão é a seguinte: rejeita-se $\mathrm{H}_{0}$ no nível de significância 
especificado $\alpha(1 \%)$, se $\mathrm{F}>\mathrm{F}_{\text {critico, }}$ caso contrário não se rejeita $\mathrm{H}_{0}$.

$\mathrm{O}$ coeficiente de determinação $\left(\mathrm{R}^{2}\right)$ é igual à soma dos quadrados devidos à regressão, dividida pela soma total dos quadrados. O coeficiente de determinação mede a proporção da variação que é explicada pela variável independente no modelo de regressão. Alguns estatísticos sugerem que seja calculado um $\mathrm{R}^{2}$ ajustado para refletir tanto o número de variáveis explicativas no modelo quanto o tamanho da amostra. O $\mathrm{R}^{2}$ ajustado é definido pela eq. (7):

$$
\mathrm{R}_{\text {ajust }}^{2}=1-\left[\left(1-\mathrm{R}^{2}\right) \frac{\mathrm{n}-1}{\mathrm{n}-2}\right]
$$

Embora o método dos mínimos quadrados resulte em uma linha que se ajusta aos dados com a quantidade mínima de variação, a equação de regressão não é um método perfeito de previsão, a menos que todos os pontos de dados estejam exatamente na linha de regressão. Esta linha serve somente para uma previsão aproximada de um valor $\mathrm{H}_{\mathrm{f}}$ para um dado valor de Q. Para tanto se usou o erro padrão da estimativa, que representa uma medida de variabilidade em torno da linha de regressão, como uma estatística para mensurar a variabilidade dos reais valores de $\mathrm{H}_{\mathrm{f}}$, através dos valores previstos de $\mathrm{H}_{\mathrm{f}}$. Para o caso da análise da perda de carga em função da vazão no filtro, o erro padrão da estimativa dada pelo símbolo $\mathrm{S}_{\mathrm{Hf} \mathrm{Q}}$ foi definido pela eq.(8):

$$
S_{\text {Hf Q }}=\sqrt{\frac{\sum_{i=1}^{n}\left(H_{f_{i}}-\hat{H}_{f_{i}}\right)^{2}}{n-2}}
$$

O erro padrão da estimativa é medido em unidades da variável dependente (m.c.a ). A interpretação do erro padrão do ajuste, então, é análogo àquela do desvio padrão.

Para a estimativa do intervalo de confiança para os coeficientes da regressão utilizou-se a estatística t ao nível de 5\% de probabilidade. Essa estatística é obtida dividindo-se o valor do coeficiente da regressão pelo erro padrão. Daí calculou-se os intervalos de confiança para os parâmetros do modelo. Assim, diz-se que os verdadeiros valores de $\hat{\beta}_{0}, \hat{\beta}_{1}$ estarão no intervalo especificado com $95 \%$ de confiança.

\section{RESULTADOS E DISCUSSÃO}

\section{Filtros de tela}

Nas Figuras 1. 2, 3 e 4 estão apresentadas as equações e curvas de perda de carga em função da vazão, obtidas em laboratório e informadas no catalogo dos fabricantes (AMIAD, 2004), par os filtros de tela de $3 / 4 ", 1$, $1^{1 / 2 "}$ e $2^{\prime \prime}$. Também são apresentadas curvas que definem o intervalo de predição ao nível de 5\% de probabilidade (linhas tracejadas). Para todos os filtros analisados em laboratório observam-se altos valores dos coeficientes de determinação, indicando uma forte relação potencial entre as variáveis vazão e perda de carga, conforme foi obtido por Testezlaf e Ramos (1995). Poe exemplo, para o filtro de tela de 3/4" obteve-se $\mathrm{R}^{2}=0,9992$, indicando que $99,92 \%$ das variações na perda de carga podem ser explicadas pela variabilidade na vazão. Isto também significa que a utilização do modelo de regressão potencial reduziu a variabilidade na precisão da perda de carga em 99,92\%. Somente $0,08 \%$ da variabilidade da perda de carga pode ser explicada por fatores ao acaso.

Nas Tabelas 3, 4, 5 e 6 apresentam-se as análises de variância da regressão para os filtros de tela 3/4", 1', $1^{1 / 2 "}$ e $2^{\prime}$. Para todos os casos pode-se rejeitar a hipótese de que não existe relação entre $\mathrm{H}_{\mathrm{f}}$ e Q, ao nível de $5 \%$ de significância, e concluir que a variável explicativa $\mathrm{Q}$ está relacionada à perda de carga pelo modelo potencial utilizado.

Nas Tabelas 7, 8, 9 e 10 apresentam-se os valores dos parâmetros do modelo de regressão $\left(\beta_{0}\right.$ e $\left.\beta_{1}\right)$ e seus limites de confiança, a $95 \%$ de probabilidade. Assim, por exemplo, para filtro de tela de $3 / 4$ ", diz-se que o verdadeiro valor de $\beta_{0}$ é estimado com $95 \%$ de confiança como estando entre 0,64436 e 0,67382 , e os valores de $\beta_{1}$ entre $1,96371 \mathrm{e}$ 2,01148 . As equações de perda de carga em função da vazão determinadas em laboratório estão representadas na Tabela 11. 


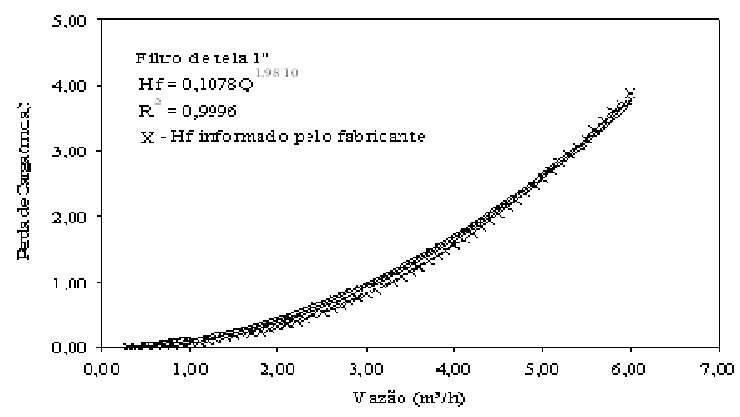

Figura 2 - Perda de carga em função da vazão para o filtro de tela de 1 ",

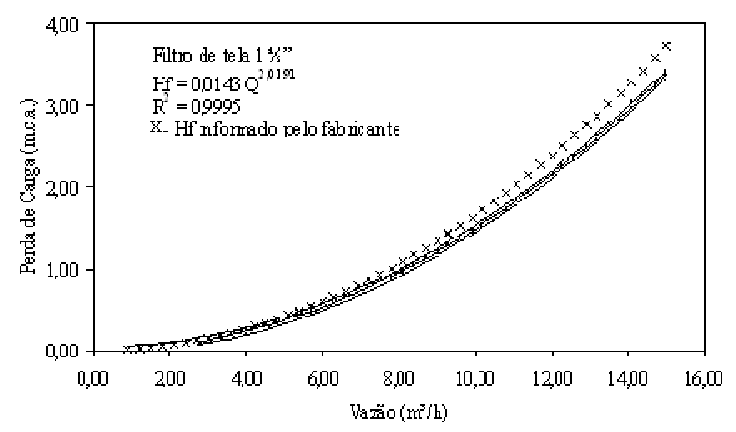

Figura 3 - Perda de carga em função da vazão para o filtro de tela de $1 \frac{1}{2}$ "

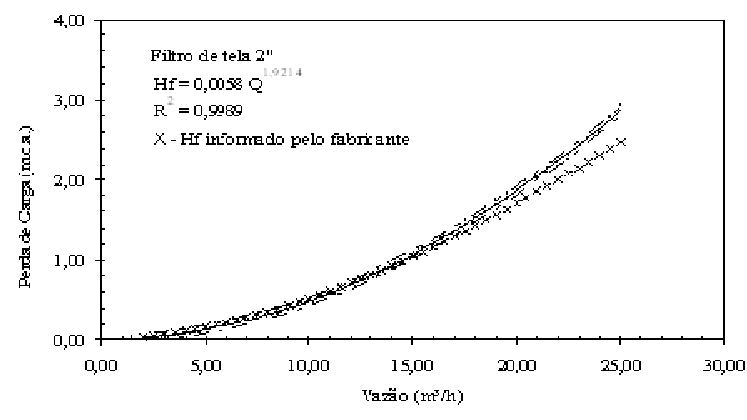

Figura 4 - Perda de carga em função da vazão para o filtro de tela de 2 "

\section{CONCLUSÕES}

As equações empíricas propostas neste trabalho que relacionam a perda de carga com a vazão, apresentaram bons resultados com coeficientes de determinação acima de $99 \%$. Desta maneira, recomenda-se a sua utilização para dimensionamento de projetos, por serem equações simples e confiáveis.

Foi possível também estudar as curvas dos gráficos $H_{\mathrm{f}}=\beta_{0} \mathrm{Q}^{\beta_{1}}$ e verificamos que a perda de carga varia diretamente proporcional à vazão em volume.

\section{REFERÊNCIAS BIBLIOGRÁFICAS}

AMIAD D. N. CHEVEL KORAZIM. Sistemas de filtrado y fertigación. Israel; 2004. 1v. (catálogo de filtros)

CAIXETA, A. V. Perda de carga em tubos e conexões de PVC utilizados em sistemas portáteis de irrigação localizada. 1991. 115p. Dissertação (Mestrado em Irrigação e Drenagem) Escola Superior de Agricultura "Luiz de Queiroz", Universidade de São Paulo, Piracicaba, 1991.

CABELLO, F. P. Riegos localizados de alta frecuencia: goteo, microaspersion, exudación. 3.ed. Madri: Mundi-Prensa, 1996. 513p.

MATSURA, E. E.; TESTEZLAF, R.; ALMEIDA NETO, J. A. Perda de carga em filtros de areia e de tela do sistema de irrigação localizada. In: XVII Congresso Brasileiro de Engenharia Agrícola (CONBEA). Recife. P.585-607, 1989.

TESTEZLAF, R.; RAMOS, J. P. S. Sistemas automatizados para determinação de perda de carga em filtros de tela e disco usados na irrigação localizada. Pesquisa Agropecuária Brasileira, Brasília. V.30, p.1079-1088, 1995. 
Tabela 3 - Análise de variância para a significância da regressão, para filtro de tela 3/4"

\begin{tabular}{|c|c|c|c|c|c|}
\hline $\begin{array}{l}\text { Fonte de } \\
\text { Variação }\end{array}$ & $\begin{array}{l}\text { Soma dos } \\
\text { Quadrados }\end{array}$ & $\begin{array}{l}\text { Graus de } \\
\text { liberdade }\end{array}$ & $\begin{array}{c}\text { Quadrado } \\
\text { Médio }\end{array}$ & $\mathrm{F}$ & $\begin{array}{c}\mathrm{F}_{\text {crítico }} \\
(\alpha=1 \%)\end{array}$ \\
\hline Regressão & 185,51256 & 1 & 185,51256 & 70200,3 & 7,01 \\
\hline Erro & 0,14384 & 56 & 0,00257 & & \\
\hline Total & 185,65640 & 57 & & & \\
\hline
\end{tabular}

Tabela 4 - Análise de variância para a significância da regressão, para filtro de tela 1"

\begin{tabular}{cccccc}
\hline Fonte de & Soma dos & Graus de & Quadrado & F & $F_{\text {crítico }}$ \\
Variação & Quadrados & liberdade & Médio & & $(\alpha, 1 \%)$ \\
\hline Regressão & 147,55965 & 1 & 147,55965 & 311250 & 6,84 \\
Erro & 0,0540459 & 114 & 0,00047 & & \\
Total & 147,6137 & 115 & & & \\
\hline
\end{tabular}

Tabela 5 - Análise de variância para a significância da regressão, para filtro de tela 1 1/2”

\begin{tabular}{cccc|cc}
\hline Fonte de & Soma dos & Graus de & Quadrado & F & $F_{\text {crítico }}$ \\
Variação & Quadrados & liberdade & Médio & & $(\alpha)$ \\
\hline Regressão & 98,96866 & 1 & 98,96866 & 203379 & 6,73 \\
Erro & 0,04574 & 94 & 0,00048 & & \\
Total & 99,01441 & 95 & & & \\
\hline
\end{tabular}

Tabela 6 - Análise de variância para a significância da regressão, para o filtro de tela 2"

\begin{tabular}{|c|c|c|c|c|c|}
\hline $\begin{array}{l}\text { Fonte de } \\
\text { Variação }\end{array}$ & $\begin{array}{l}\text { Soma dos } \\
\text { Quadrados }\end{array}$ & $\begin{array}{l}\text { Graus de } \\
\text { liberdade }\end{array}$ & $\begin{array}{l}\text { Quadrado } \\
\text { Médio }\end{array}$ & $\mathrm{F}$ & $\begin{array}{c}\mathrm{F}_{\text {crítico }} \\
(\alpha=1 \%)\end{array}$ \\
\hline Regressão & 70,59213 & 1 & 70,59213 & 91856,6 & 6,72 \\
\hline Erro & 0,07147 & 93 & 0,00077 & & \\
\hline Total & 70,66360 & 94 & & & \\
\hline
\end{tabular}

Tabela 7 - Intervalos de confiança para parâmetros da regressão, para o filtro de tela 3/4"

\begin{tabular}{ccccc} 
Coeficientes & Valor & Erro Padrão & Valor $-\mathrm{t}$ & Intervalos de Confiança 95\% \\
$\beta 0$ & 0,65908 & 0,00735 & 89,62425 & $0,64436 \leftrightarrow 0,67382$ \\
$\beta 1$ & 1,98759 & 0,01192 & 166,67734 & $1,96371 \leftrightarrow 2,01148$ \\
\hline
\end{tabular}

Tabela 8 - Intervalos de confiança para parâmetros da regressão, para o filtro de tela 1"

\begin{tabular}{ccccc} 
Coeficientes & Valor & Erro Padrão & Valor $-\mathrm{t}$ & Intervalos de Confiança 95\% \\
$\beta 0$ & 0,10787 & 0,00099 & 108,21713 & $0,10589 \leftrightarrow 0,10984$ \\
$\beta 1$ & 1,98101 & 0,00573 & 345,41117 & $1,96965 \leftrightarrow 1,99237$ \\
\hline
\end{tabular}

Tabela 9 - Intervalos de confiança para parâmetros da regressão, para o filtro de tela 1 1⁄2"

\begin{tabular}{ccccc} 
Coeficientes & Valor & Erro Padrão & Valor $-\mathrm{t}$ & Intervalos de Confiança 95\% \\
$\beta 0$ & 0,01428 & 0,00026 & 54,99162 & $0,01376 \leftrightarrow 0,01479$ \\
$\beta 1$ & 2,01905 & 0,00719 & 280,60134 & $2,00476 \leftrightarrow 2,03333$ \\
\hline
\end{tabular}

Tabela 10 - Intervalos de confiança para parâmetros da regressão, para o filtro de tela 2"

\begin{tabular}{ccccc} 
Coeficientes & Valor & Erro Padrão & Valor $-\mathrm{t}$ & Intervalos de Confiança 95\% \\
$\beta 0$ & 0,00589 & 0,00018 & 32,74732 & $0,00554 \leftrightarrow 0,00625$ \\
$\beta 1$ & 1,92142 & 0,01008 & 190,60651 & $1,90141 \leftrightarrow 1,94144$ \\
\hline
\end{tabular}

Tabela 11 - Equações de Perda de carga $\left(\mathrm{H}_{\mathrm{f}}\right)$ dos filtros de tela

\begin{tabular}{ccccc}
\hline Tipo & Modelo & $\mathrm{N}^{\circ}$ de mesh & $\mathrm{R}^{2}$ & Equacão \\
Tela & 3/4": & 120 & 0,9992 & $\mathrm{Hf}=0,6591 \mathrm{Q}^{1,9875}$ \\
Tela & 1 "” & 120 & 0,9996 & $\mathrm{Hf}=0,1078 \mathrm{Q}^{1,9810}$ \\
Tela & 1 1/2": & 120 & 0,9995 & $\mathrm{Hf}=0,0143 \mathrm{Q}^{2,0191}$ \\
Tela & $2 "$ & 120 & 0,9989 & $\mathrm{Hf}=0,0058 \mathrm{Q}^{1,9214}$
\end{tabular}

